\title{
First-principles calculations of ${ }^{17} \mathrm{O}$ NMR chemical shielding in $\mathrm{Pb}\left(\mathrm{Zr}_{1 / 2} \mathrm{Ti}_{1 / 2}\right) \mathrm{O}_{3}$ and $\mathrm{Pb}\left(\mathrm{Mg}_{1 / 3} \mathrm{Nb}_{2 / 3}\right) \mathrm{O}_{3}$ : linear dependence on transition-metal/oxygen bond lengths
}

\author{
Daniel L. Pechkis, Eric J. Walter and Henry Krakauer \\ Department of Physics, College of William and Mary, Williamsburg, VA 23187-8795.
}

(Dated: October 6, 2018)

\begin{abstract}
First-principles density functional theory (DFT) oxygen chemical shift tensors were calculated for $\mathrm{A}\left(\mathrm{B}, \mathrm{B}^{\prime}\right) \mathrm{O}_{3}$ perovskite alloys $\mathrm{Pb}\left(\mathrm{Zr}_{1 / 2} \mathrm{Ti}_{1 / 2}\right) \mathrm{O}_{3}(\mathrm{PZT})$ and $\mathrm{Pb}\left(\mathrm{Mg}_{1 / 3} \mathrm{Nb}_{2 / 3}\right) \mathrm{O}_{3}$ ( $\left.\mathrm{PMN}\right)$. Quantum chemistry methods for embedded clusters and the GIPAW method [C. J. Pickard and F. Mauri, Phys. Rev. B 63245101 (2001)] for periodic boundary conditions were used. Results from both methods are in good agreement for PZT and prototypical perovskites. PMN results were obtained using only GIPAW. Both isotropic $\delta_{\text {iso }}$ and axial $\delta_{\text {ax }}$ chemical shifts were found to vary approximately linearly as a function of the nearest-distance transition-metal/oxygen bond length, $r_{\mathrm{s}}$. Using these results, we argue against Ti clustering in PZT, as conjectured from recent ${ }^{17} \mathrm{O}$ NMR magic-angle-spinning measurements. Our findings indicate that ${ }^{17} \mathrm{O}$ NMR measurements, coupled with first-principles calculations, can be an important probe of local structure in complex perovskite solid solutions.
\end{abstract}

PACS numbers: 76.60.Cq 77.84.-s

\section{INTRODUCTION}

High performance solid solution ferroelectrics, based on the ideal $\mathrm{ABO}_{3}$ perovskite structure, are widely used in technological applications such as ultrasonic transducers, sensors, actuators, and thin film applications $\frac{1.2}{.2}$ The strong electromechanical coupling in these materials is related to a balance of competing instabilities, such as cation off-centerings and oxygen octahedral rotations. The perovskite structure offers many ways to fine tune these interactions through chemical substitutions, such as alloying on the A and/or B sites, and through epitaxial control in layered and thin film geometries $\underline{\underline{1}}$

Solid state nuclear magnetic resonance (NMR) has increasingly been used to study the local structure and dynamics of these complex perovskites ${ }^{3-6}-\underline{n}$ NMR spectra of a target nucleus are largely determined by the coupling of its magnetic dipole and electric quadrupole moments with the local magnetic field and electric field gradient, respectively. The interpretation of NMR spectra in complex solid solution perovskites is complicated by the presence of broad spectral features due to disorder. First-principles calculations of electric field gradient $\underline{\underline{7}}^{\underline{T}}$ and chemical shielding $\stackrel{8}{8}$ tensors can play an important role interpreting NMR spectra in these materials.

Previously we demonstrated a near linear dependence of the chemical shielding tensor $\hat{\sigma}$ on the oxygen nearest neighbor $\mathrm{B}-\mathrm{O}$ bond distance $r_{\mathrm{s}}$ for the prototypical perovskites $\mathrm{BaTiO}_{3}(\mathrm{BT}), \mathrm{SrTiO}_{3}(\mathrm{ST}), \mathrm{PbTiO}_{3}(\mathrm{PT})$,

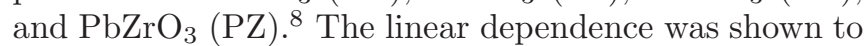
arise from large paramagnetic contributions to $\sigma_{x}$ and $\sigma_{y}$ principal values (our convention identifies the " $z$ " principal axis as that most nearly parallel to the B-O-B bond direction), due to virtual transitions between $\mathrm{O}(2 \mathrm{p})$ and unoccupied $\mathrm{B}(n \mathrm{~d})$ states. This linear variation is confirmed here for two complex perovskite solid solutions, PZT and PMN.
First-principles chemical shielding calculations have traditionally been done with the embedded cluster approach, using standard quantum chemistry methods ${ }^{9}-11$ with gaussian type orbitals (GTO). More recently, the planewave based GIPAW method with PBC has provided an alternative approach. Relatively few calculations for transition metal oxides have been reported using either technique. Here we use complementary calculations with both methods to cross validate convergence with respect to cluster size and termination effects, basis sets, and the accuracy of pseudopotentials (PSPs).

Quantum-chemistry methods can calculate chemical shielding tensors for embedded clusters, using a range of approximations, from Hartree Fock and density functional theory (DFT) with semilocal or hybrid exchangecorrelation functionals, to explicitly correlated methods such Moller-Plesset perturbation theory and coupled cluster approaches 12 -14 The principal difficulties with the embedded cluster approach are controlling size and basis set convergence. Size effects can be monitored by studying increasingly larger clusters. Long-range electrostatic interactions can be handled by embedding the cluster in large arrays of point charges and eliminating depolarizing fields, as described in Ref. 8. Achieving the basis set limit can be problematic in some cases, because atom-centered GTO's do not form a complete orthogonal basis. Nevertheless, basis set convergence is generally well controlled through the use of standardized GTO basis sets $\underline{15}$ The cluster approach becomes inefficient, however, for complex systems, because separate cluster calculations are usually required for each inequivalent target atom.

GIPAW calculations are naturally adapted to ordered crystalline solids, since size convergence is effectively achieved by using primitive unit cells with wellconverged $k$-point quadrature grids for Brillouin zone integrations. Disordered solids can be treated using supercells. Planewaves form a complete basis, so convergence 
to the basis set limit is straightforward. The method applies the projector-augmented-wave (PAW) reconstruction $\underline{16}$ to a conventional PSP calculation to obtain allelectron valence wave functions, which are required for accurate calculations of the chemical shielding. There are two principle issues with the GIPAW method. GIPAW PSPs are more difficult to construct than standard norm-conserving PSPs. To achieve good transferability, they may require multiple partial wave channels and large planewave cutoff energies for some target atoms. The construction of the PAW atomic-like augmentation basis also requires care. To date, GIPAW calculations have been carried out for only a limited number of transition metal oxide systems $\underline{\underline{17}, 18}$

PZT is a homovalent mixture of $\mathrm{Ti}^{4+}$ and $\mathrm{Zr}^{4+}$ transition metal cations, while PMN is a heterovalent 2:1 mixture of the $\mathrm{Nb}^{5+}$ transition metal cation and the $\mathrm{Mg}^{2+}$ alkaline earth cation. Both embedded cluster and GIPAW calculations were carried out for PZT, while PMN results were obtained only with the GIPAW method, using PBC. First-principles relaxed structural models were used to simulate PZT and PMN structures.

The remainder of the paper is organized as follows. The theoretical approaches are described in Sec. [II Results and discussion are presented in Secs. [II] and IV] respectively. We summarize and conclude in Sec. V.

\section{THEORETICAL METHODS}

The chemical shielding tensor $\hat{\sigma}$ determines the total magnetic field at an atomic nucleus,

$$
\mathbf{B}=(1-\hat{\sigma}) \mathbf{B}_{\mathrm{ext}},
$$

where $\mathbf{B}_{\text {ext }}$ is the external field. For the systems considered here, $\hat{\sigma}$ is calculated using embedded cluster and GIPAW-PBC methods. The symmetric $\underline{19,20} \hat{\sigma}$ tensor is determined by its principal axis components, with isotropic and anisotropic parts, conventionally defined $\mathrm{as}^{21}$

$$
\begin{gathered}
\sigma_{\text {iso }}=\frac{1}{3}\left(\sigma_{x}+\sigma_{y}+\sigma_{z}\right)=\frac{1}{3} \operatorname{Tr} \hat{\sigma} \\
\sigma_{\text {ax }}=\frac{1}{6}\left(2 \sigma_{z}-\sigma_{x}-\sigma_{y}\right)=\frac{1}{2}\left(\sigma_{z}-\sigma_{\text {iso }}\right) \\
\sigma_{\text {aniso }}=\frac{1}{2}\left(\sigma_{y}-\sigma_{x}\right)
\end{gathered}
$$

As mentioned, our convention for the perovskite structure identifies the " $z$ " principal axis as that most nearly parallel to the B-O-B bond direction. NMR measurements of $\hat{\sigma}$ are usually reported with respect to a reference material, where the chemical shift tensor $\hat{\delta}$ is defined as 13

$$
\hat{\delta}=-\left(\hat{\sigma}-\sigma_{\text {ref }}\right),
$$

with corresponding definitions to those in Eq. 2 The experimental reference for oxygen is liquid $\mathrm{H}_{2} \mathrm{O}$.

The theoretical oxygen reference value $\sigma_{\text {ref }}^{\text {th. }}$ is determined from a linear regression of $\delta_{\text {iso }}^{\text {expt. }}$ versus $\sigma_{\text {iso }}^{\text {theory }}, \underline{18,22}$
This yields the relation

$$
\delta^{\text {th. }}=-m\left(\sigma^{\text {th. }}-\sigma_{\text {ref }}^{\text {th. }}\right) .
$$

Rather than regarding the slope $m$ as an independent fitting parameter, we set $m=1$, which leaves $\sigma_{\text {ref }}^{\text {th. }}$ as the only independent parameter. Allowing $m$ to vary yields fits of similar overall quality, but somewhat distorts chemical shift differences between inequivalent oxygen sites within the same material. Constraining $m=1$ allows better cancellation of errors, due to systematic effects such as the choice of methodological approach or DFT exchange-correlation functional.

\section{A. Embedded cluster calculations}

A detailed discussion of this approach is given in Ref. 8. We briefly summarize some of the key features of this method. With central $\mathrm{O}$ atoms, embedded clusters consisted of either 21 "quantum" (QM) atoms, $\left(\mathrm{A}_{4} \mathrm{~B}_{2} \mathrm{O}_{15}\right)^{14-}$, or $65 \mathrm{QM}$ atoms, $\left(\mathrm{A}_{4} \mathrm{~B}_{10} \mathrm{O}_{51}\right)^{51-}$. In these clusters, all cation atoms are fully coordinated with QM O atoms. The QM clusters are further surrounded by a large array of point charges, which reproduce the Madelung potential in the QM region $\underline{\underline{23}}$ To alleviate artificial polarization of boundary $\mathrm{O}(2 \mathrm{p})$ states, the nearestneighbor (nn) and the next-nearest-neighbor cation point charges of boundary O atoms are replaced by "empty" PSPs (ePSPs) $\stackrel{8,24}{=}$ Finally, in non-centrosymmetric clusters, depolarizing electric fields are removed by applying an external electric field $\stackrel{\underline{8}}{\underline{ }}$

Calculations were performed with the GAUSSIAN computational package,,$\frac{9.11}{1}$ and the chemical shielding tensor was determined using the continuous set of gauge transformations (CSGT) method, $\stackrel{25,26}{ }$ Calculations were done using the DFT hybrid B3LYP 27 , as well as generalized gradient approximations (GGA), using the PW91 ${ }^{28}$ and $\mathrm{PBE}^{29}$ forms. Douglas-Kroll-Hess 2nd order scalarrelativistic calculations were performed on selected systems. Atom-centered GTO basis functions were associated with all the QM atoms. All-electron treatments were used for the $\mathrm{O}$ and $\mathrm{Ti}$ atoms, while the other QM atoms were represented using scalar-relativistic small core (scalar-RSC) PSPs [also called effective core potentials (ECPs)]. The well-converged GTO basis sets and ECPs used for these calculations are described in Ref. 8 and were taken from the EMSL website $\frac{15}{}$

\section{B. GIPAW calculations}

Calculations with PBC used the GIPAW functionality of the QuantumESPRESSO (QE) code ${ }^{30}$ These calculations are done in two steps. A standard ground state norm-conserving PSP calculation is first performed. This is followed by a linear response calculation in the presence of an external magnetic field. The linear response 
TABLE I: Pseudopotential construction parameters, see text for description.

\begin{tabular}{|c|c|c|c|c|}
\hline & reference state & $\mathrm{r}_{c}(\mathrm{au})$ & reference energies (Ry) & core correction radius $(\mathrm{au})$ \\
\hline$\overline{\mathrm{O}}$ & $2 \mathbf{s}^{2}, 2 \mathrm{p}^{6}, 3 d, 3 s, 3 p$ & $1.2,1.2,1.5$ & $*{ }^{*}, 0.10,0.10,0.10$ & 0.34 \\
\hline $\mathrm{Ti}$ & $3 \mathrm{~s}^{2}, 3 \mathrm{p}^{6}, 3 \mathrm{~d}^{0}, 4 s, 4 p, 4 d$ & $0.9,0.9,0.9$ & $*, *, *,-2.73,0.50,0.10$ & 0.53 \\
\hline $\mathrm{Pb}$ & $6 \mathrm{~s}^{2}, 6 \mathrm{p}^{0}, 5 \mathrm{~d}^{10}, 7 s, 7 p, 6 d$ & $2.0,2.4,1.0$ & $*, *, *,-1.00,-0.20,-1.80$ & 0.89 \\
\hline $\mathrm{Zr}$ & $4 \mathrm{~s}^{2}, 4 \mathrm{p}^{6}, 4 \mathrm{~d}^{0}, 5 s, 5 p, 5 d$ & $1.0,1.2,1.4$ & $*, * *,-2.45,-2.00,-1.37$ & 0.80 \\
\hline $\mathrm{Sr}$ & $4 \mathrm{~s}^{2}, 4 \mathbf{p}^{6}, 4 \mathrm{~d}^{0}, 5 s, 5 p$ & $1.2,1.3,1.7$ & $*, *,-1.00,-0.72$ & 0.88 \\
\hline $\mathrm{Ba}$ & $5 \mathrm{~s}^{2}, 5 \mathrm{p}^{6}, 5 d, 6 s, 6 p$ & $1.5,1.7,2.0$ & $*, *,-0.95,-0.90,-1.50$ & 1.19 \\
\hline $\mathrm{Mg}$ & $2 \mathrm{~s}^{2}, 2 \mathrm{p}^{6}, 3 d, 3 s, 3 p$ & $0.6,0.6,1.5$ & $*, *, 0.3,-1.3,0.1$ & 0.30 \\
\hline $\mathrm{Nb}$ & $4 \mathrm{~s}^{2}, 4 \mathrm{p}^{6}, 4 \mathrm{~d}^{0}, 5 s, 5 p, 5 d$ & $1.0,1.1,1.3$ & $*, *, *,-3.30,-2.70,-1.27$ & 0.80 \\
\hline $\mathrm{K}$ & $3 \mathrm{~s}^{2}, 3 \mathrm{p}^{6}, 3 \mathrm{~d}^{0}, 4 s, 4 p$ & $1.4,1.5,1.5$ & $*, *, *,-1.50,-0.80$ & 0.50 \\
\hline
\end{tabular}

calculation uses all-electron like valence wave functions, which are represented by planewaves, modified near the nuclei by atomic-like PAW augmentation basis functions. The PAW basis functions are constructed when the PSP is generated, as further discussed below. Calculations reported below used well converged Monkhorst-Pack ${ }^{31}$ Brillouin zone $k$-point sampling, e.g. $6 \times 6 \times 6$ for the prototypical perovskites ST, BT, and PT. Unless otherwise specified, the PBE GGA functional was used in all QE calculations.

All PSPs were constructed using the "ld1" PSP generation code (distributed with QE). All norm-conserving PSPs were scalar-relativistic Troullier-Martins ${ }^{32}$ type. Table I shows the construction parameters used for all PSPs employed in this paper. The local channel is indicated by boldface type. States that are in italics were generated using a Hamann type 33 reference state. The $r_{c}$ 's correspond to the $s, p$, and $d$ channels respectively. The next column shows the PSP reference energies for the $s, p$, and $d$ channels. The symbol ' $*$ ' indicates that the corresponding all-electron eigenvalue was used for this state. It should be noted that these parameters are for the PSP projectors used in the ground state selfconsistent total-energy calculations. Parallel to the PSP construction, a second atomic calculation is performed to generate the required GIPAW augmentation basis functions, consisting of all-electron and pseudo partial wave radial functions. The number of GIPAW angular momentum channels was the same as for the PSPs. For all except $\mathrm{Pb}, \mathrm{O}$ and $\mathrm{K}$, the augmentation basis functions were generated using the corresponding all-electron eigenvalues (n.b., the $\mathrm{Ti}^{4+} 4 d$ state is bound, for example), rather than the values in Table \. For $\mathrm{Pb}, \mathrm{O}$ and $\mathrm{K}$, the values in Table $\Pi$ were used, except for the $\mathrm{Pb} 7 \mathrm{~s}$, which used the all-electron eigenvalue. The final column shows the non-linear core-correction radius for each potential. A conservative 350 Ry energy cutoff was used. This could have been reduced by using larger $r_{c}$ 's for the metal PSPs. The 350 Ry energy cutoff, while high, was easily tractable for all systems studied in this paper. This cutoff yields chemical shieldings to within about $1 \mathrm{ppm}$, as indicated by test calculations with other settings.
TABLE II: Comparison of chemical shielding results, using embedded clusters and GIPAW-PBC from QE and Ref. 18. The principle values of the oxygen chemical shielding tensor are presented for the $\mathrm{TiO}_{2}$ molecule and rutile, cubic ST, cubic BT, and tetragonal PT. All values are from GGA calculations. Embedded cluster results are labeled as C- $n$, where $n$ is is the number of QM atoms in the cluster. The GIPAW-PBC and $\mathrm{C}-65$ calculations were done with relativistic PBE, while the C-21 calculations were done with non-relativistic PW91.

\begin{tabular}{|c|c|c|c|c|}
\hline & $\sigma_{x}$ & $\sigma_{y}$ & $\sigma_{z}$ & $\sigma_{\text {iso }}$ \\
\hline \multicolumn{5}{|c|}{$\overline{\mathrm{TiO}_{2} \text { (molecule) }}$} \\
\hline Gaussian & -1803 & -801 & 148 & -819 \\
\hline GIPAW & -1826 & -811 & 146 & -830 \\
\hline \multicolumn{5}{|c|}{$\overline{\mathrm{TiO}_{2} \text { (rutile) }}$} \\
\hline C-99 & -499 & -380 & -306 & -395 \\
\hline GIPAW & -483 & -380 & -296 & -386 \\
\hline \multicolumn{5}{|c|}{$\mathrm{SrTiO}_{3}$ (cubic) } \\
\hline C-21 & -353 & -353 & 46 & -220 \\
\hline C-65 & -403 & -403 & 27 & -260 \\
\hline GIPAW & -429 & -429 & 7 & $-284 /-287^{a}$ \\
\hline \multicolumn{5}{|c|}{$\mathrm{BaTiO}_{3}$ (cubic) } \\
\hline $\mathrm{C}-21$ & -414 & -414 & 49 & -260 \\
\hline C-65 & -483 & -483 & 22 & -315 \\
\hline GIPAW & -529 & -529 & -31 & $-363 /-379^{b}$ \\
\hline \multicolumn{5}{|c|}{$\mathrm{PbTiO}_{3}$-axial O (P4mm) } \\
\hline $\mathrm{C}-21$ & -562 & -562 & 123 & -334 \\
\hline C-65 & -599 & -599 & 67 & -377 \\
\hline GIPAW & -630 & -630 & 77 & -394 \\
\hline \multicolumn{5}{|c|}{$\mathrm{PbTiO}_{3}$-equatorial $\mathrm{O}(\mathrm{P} 4 \mathrm{~mm})$} \\
\hline C-21 & -286 & -228 & -32 & -182 \\
\hline C-65 & -365 & -277 & -35 & -226 \\
\hline GIPAW & -398 & -284 & -23 & -235 \\
\hline
\end{tabular}

${ }^{a}$ Ref. 18 determined $\sigma_{i s o}$ using the experimental structure

${ }^{b}$ Ref. 18 determined $\sigma_{\text {iso }}$ using the relaxed structure

\section{Comparison of methods}

Table II shows the comparison between the GIPAW and cluster approach for the $\mathrm{TiO}_{2}$ molecule, the rutile crystalline solid, as well as some results for prototypical 
TABLE III: The derived theoretical oxygen reference value, $\sigma_{\text {ref }}^{\text {th. }}$, is used to determine theoretical isotropic chemical shifts from the corresponding calculated isotropic chemical shielding values. Experimental chemical shifts are shown for comparison. The values of $\sigma_{\text {ref }}^{\text {th }}$ are shown in the third row from the bottom of this table. The rms error and maximum deviation of the calculated shifts, compared to experiment, are also shown. Calculated cluster results (21 and 65 QM atoms) with B3LYP and PW91 exchange-correlation are shown together with GIPAW-PBC with PBE exchange-correlation. (PZ experimental chemical shift site assignments were corrected in Ref. 8 and are used here.)

\begin{tabular}{|c|c|c|c|c|c|c|c|c|c|}
\hline & & \multirow[t]{3}{*}{$\overline{\operatorname{Expt}^{a}}$} & \multicolumn{4}{|c|}{ Cluster } & \multicolumn{3}{|c|}{ 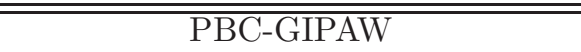 } \\
\hline & & & \multicolumn{2}{|c|}{ B3LYP } & \multicolumn{2}{|c|}{ PW91 } & \multirow[t]{2}{*}{ present $^{f}$} & \multirow[t]{2}{*}{ present } & \multirow[t]{2}{*}{ other } \\
\hline & & & 21 & 65 & 21 & 65 & & & \\
\hline ST (cubic) & & $467 \pm 5$ & 491 & 477 & 494 & 491 & 491 & 496 & $503^{b}$ \\
\hline BT (cubic) & & $546 \pm 5$ & 536 & 537 & 534 & 547 & 570 & 575 & $595^{c}$ \\
\hline \multicolumn{10}{|l|}{$\mathrm{BT}(\mathrm{P} 4 \mathrm{~mm})$} \\
\hline & $\mathrm{O}_{\mathrm{ax}}$ & $570 \pm 5$ & 591 & & 571 & & & 579 & $573^{d} / 611^{e}$ \\
\hline & $\mathrm{O}_{\mathrm{eq}}$ & $520 \pm 5$ & 515 & & 516 & & & 535 & $563^{d} / 531^{e}$ \\
\hline & $\Delta\left(\mathrm{O}_{\mathrm{ax}}-\mathrm{O}_{\mathrm{eq}}\right)$ & 50 & 76 & & 56 & & & 44 & $10 / 80$ \\
\hline \multicolumn{10}{|l|}{$\mathrm{PT}(\mathrm{P} 4 \mathrm{~mm})$} \\
\hline & $\mathrm{O}_{\mathrm{ax}}$ & $647 \pm 2$ & 644 & 644 & 608 & 609 & 601 & 606 & \\
\hline & $\mathrm{O}_{\mathrm{eq}}$ & $443 \pm 2$ & 449 & 445 & 457 & 455 & 442 & 447 & \\
\hline & $\Delta\left(\mathrm{O}_{\mathrm{ax}}-\mathrm{O}_{\mathrm{eq}}\right)$ & 204 & 195 & 199 & 152 & 154 & 159 & 159 & \\
\hline \multicolumn{10}{|l|}{ PZ (Pbam) } \\
\hline & $\mathrm{O} 1-4 \mathrm{~g}$ & $365 \pm 2$ & 357 & & 367 & & & 355 & \\
\hline & $\mathrm{O} 1^{\prime}-4 \mathrm{~g}$ & $351 \pm 2$ & 346 & & 356 & & & 336 & \\
\hline & $\mathrm{O} 2-8 \mathrm{i}$ & $356 \pm 2$ & 355 & & 364 & & & 349 & \\
\hline & O3-4f & $329 \pm 2$ & 324 & & 336 & & & 309 & \\
\hline & $\mathrm{O} 4-4 \mathrm{e}$ & $408 \pm 2$ & 392 & & 399 & & & 415 & \\
\hline & $\Delta(\mathrm{O} 4-\mathrm{O} 1)$ & 43 & 35 & & 31 & & & 60 & \\
\hline & $\Delta\left(\mathrm{O} 4-\mathrm{O} 1^{\prime}\right)$ & 57 & 47 & & 43 & & & 79 & \\
\hline & $\Delta \mathrm{O} 4-\mathrm{O} 2)$ & 52 & 38 & & 35 & & & 66 & \\
\hline & $\Delta(\mathrm{O} 4-\mathrm{O} 3)$ & 79 & 68 & & 63 & & & 105 & \\
\hline$\overline{\sigma_{\text {ref }}^{\text {th. }}}$ & & $288^{e}$ & 293 & 238 & 275 & 217 & 207 & 212 & 216 \\
\hline RMS error & & & 12 & 7 & 16 & 23 & 28 & 20 & 26 \\
\hline $\operatorname{Max} \operatorname{Dev}^{f}$ & & & 24 & 10 & 39 & 38 & 46 & 41 & \\
\hline
\end{tabular}

${ }^{a} \mathrm{ST}$ and BT experimental chemical shift values are from Ref. 6 and the PT and PZ experimental chemical shift values are from Ref. 4 .

${ }^{b}$ Ref. 18 determined $\sigma_{i s o}$ using the experimental structure

${ }^{c}$ Ref. 18 determined $\sigma_{\text {iso }}$ using the relaxed structure

${ }^{d}$ The ${ }^{17} \mathrm{O}$ experimental chemical shielding reference is liquid water, $\sigma_{\text {iso }}^{\text {water }}=287.5 \mathrm{ppm}^{34}$

${ }^{e} \operatorname{Max} \operatorname{Dev}=\max \left(\operatorname{abs}\left(\delta^{\text {theory }}-\delta^{\text {expt. }}\right)\right)$

${ }^{f}$ Used $\sigma_{\text {ref }}^{\text {th. }}$ determined from linear regression on the same systems as the 65 QM atom cluster-PW91 results (see text).

perovskites. Where available, published GIPAW results from Ref. 18 are also given for comparison.

$\mathrm{TiO}_{2}$ molecule calculations were for a Ti-O bond length of $1.651 \AA$ and a O-Ti-O angle of $114.2^{\circ}$. With PBCGIPAW, a $22 \times 24 \times 28$ Bohr supercell was used. The Gaussian calculation was for the isolated molecule and used a basis set of cc-pwCVQZ-DK and IGLO-III for $\mathrm{Ti}$ and $\mathrm{O}$, respectively. The experimental rutile structure was used for solid $\mathrm{TiO}_{2} \stackrel{35}{ }$ The PBC-GIPAW calculation used a $k$-point sampling of $4 \times 4 \times 6$. The cluster method used a $\mathrm{O}_{77} \mathrm{Ti}_{22}$ QM cluster with cc-pwCVTZ-DK and $6-311 \mathrm{G}(2 \mathrm{~d}, \mathrm{p})$ basis sets for the inner 3 and outer 19 Ti atoms, respectively; IGLO-III and IGLO-II basis sets were used for the inner 15 and outer $52 \mathrm{O}$ atoms, respectively. (Embedding with ePSPs and point charges was done as described above.)

Calculations for the prototypical perovskites in Table [I] used the experimental structures as described in Ref. 8 . PBC-GIPAW used a $k$-point sampling of $6 \times 6 \times 6$. Embedded clusters were constructed as above (Section $\amalg$ A), 
and results are given for 21 and 65 QM atom clusters. The $21 \mathrm{QM}$ atom cluster results are from non-relativistic calculations using the PW91 DFT functional. The 65 QM atom clusters are from scalar relativistic PBE calculations. Differences between PW91 and PBE (not shown in the Table) and relativistic effects are small, as expected. Tests on the $65 \mathrm{QM}$ atom ST cluster show that non-relativistic PBE produced $\sigma_{x, y}$ and $\sigma_{z}$ values that are $\simeq 6$ and $1.5 \mathrm{ppm}$ more positive, respectively, than PW91. Adding scalar-relativistic effects changed $\sigma_{x, y}$ and $\sigma_{z}$ by $\simeq+12$ and $+3 \mathrm{ppm}$, respectively, independent of the GGA functional. These effects are negligible for the corresponding chemical shifts, as expected, due to cancellation of errors.

The ST and PT $65 \mathrm{QM}$ atom relativistic PBE $\sigma_{x, y}$ and $\sigma_{z}$ are in good agreement with $\mathrm{PBC}$ values, differing at most by $\simeq 33$ and $20 \mathrm{ppm}$ respectively. Isotropic values for both systems are within $24 \mathrm{ppm}$ of PBC-GIPAW. A larger discrepancy is seen in BT, where the cluster values are rigidly shifted by about $+50 \mathrm{ppm}$. Size effects between the 21 and $65 \mathrm{QM}$ atom results are evident in the table. The $21 \mathrm{QM}$ atom values are more shielded [i.e., more positive (see Eq. (10)] than either the $65 \mathrm{QM}$ atom values or the PBC methods. Rigidly shifting all $21 \mathrm{QM}$ atom brings these into better agreement, indicating good cancellation of errors. This is evident in the chemical shifts shown in Table ЏII in the next section, where 21 and $65 \mathrm{QM}$ atom clusters are seen to give nearly identical chemicals shifts.

These results demonstrate that the embedded cluster and PBC-GIPAW approaches produce comparable agreement with measured isotropic chemical shifts, regardless of cluster size and methodology. Differences due to cluster size, DFT functionals, relativistic effects, and PSPs largely cancel in the chemical shifts, i.e., they are absorbed in the constant chemical shielding reference value $\sigma_{\text {ref }}^{\text {th. }}$.

\section{RESULTS}

In this section, we first describe the calculation of the theoretical oxygen chemical shielding reference $\sigma_{\text {ref }}^{\text {th }}$ [Eq. (4)]. As mentioned, this is done using linear regression of the calculated chemical shieldings with the corresponding measured chemical shifts, where available $\underline{\underline{18,22}}$ We next present calculated ${ }^{17} \mathrm{O}$ chemical shifts for two perovskite-based B-site alloys, PZT and PMN, using the derived values of $\sigma_{\text {ref }}^{\text {th }}$.

\section{A. Determination of the theoretical ${ }^{17} \mathrm{O}$ chemical shielding reference}

A linear regression was separately evaluated for the

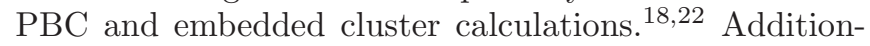
ally for the clusters, separate regressions were performed for different cluster sizes and DFT functionals. Table III shows derived $\sigma_{\text {ref }}^{\text {th. }}$ values for each case, along with the rms error and maximum deviation in the isotropic chemical shifts. The table compares the resulting calculated isotropic chemical shifts to the measured values. As in Ref. 8, experimental structures were used for all systems except PZ. For PZ, experimental lattice parameters from neutron scattering measurements were used together with internal coordinates determined from firstprinciples calculations $\underline{\underline{36}}$

Calculated cluster results (21 an 65 QM atoms) with B3LYP and PW91 exchange-correlation are shown together with GIPAW-PBC with PBE exchangecorrelation. The last column shows other GIPAW results, where available. For the $65 \mathrm{QM}$ clusters, $\sigma_{\text {ref }}^{\text {th. }}$ is derived from a more limited set of calculations, as shown in the Table. For comparison, the effect of this to be only a few ppm. B3LYP results are seen to give slightly better agreement with experiment. Using the same exchangecorrelation treatment, both the 21 and 65 atom QM clusters are seen to give nearly identical chemicals shifts. The values of $\sigma_{\text {ref }}^{\text {th. }}$ in Table $\amalg I I$ are used below to determine the theoretical chemical shifts for PZT and PMN in Tables IV and V Given the small difference between the PW91 and PBE GGA functionals and cancellation effects in chemical shifts, both PW91 and PBE are labeled as GGA in all further results below.

\section{B. Results for $\mathrm{Pb}\left(\mathrm{Zr}_{1-x} \mathrm{Ti}_{x}\right) \mathrm{O}_{3}(\mathrm{PZT})$ and $\mathrm{Pb}\left(\mathrm{Mg}_{1 / 3} \mathrm{Nb}_{2 / 3}\right) \mathrm{O}_{3}(\mathrm{PMN})$}

PZT is a homovalent mixture of $\mathrm{Ti}^{4+}$ and $\mathrm{Zr}^{4+}$ transition metal cations, while $\mathrm{Pb}\left(\mathrm{Mg}_{1 / 3} \mathrm{Nb}_{2 / 3}\right) \mathrm{O}_{3}(\mathrm{PMN})$ is a heterovalent 2:1 mixture of the $\mathrm{Nb}^{5+}$ transition metal cation and the $\mathrm{Mg}^{2+}$ alkaline earth cation. Both embedded cluster and GIPAW calculations were carried out for PZT, while PMN results were obtained only with the GIPAW method, using PBC.

Disordered PZT 50/50 was modeled using firstprinciples relaxed structures ${ }^{\underline{7}}$ with different imposed B-site ordering and symmetry: i) [001] ordering parallel to the ferroelectric polarization, with tetragonal $\mathrm{P} 4 \mathrm{~mm}$ symmetry $(\mathrm{a} \times \mathrm{a} \times 2 \mathrm{c} ; \mathrm{c} / \mathrm{a}=1.045)$; ii $)$ [001] ordering perpendicular to the [100] ferroelectric polarization, with orthorhombic P2mm symmetry $\left(\mathrm{a}^{\prime} \times \mathrm{a} \times 2 \mathrm{a} ; \mathrm{a}^{\prime} / \mathrm{a}=1.04\right)$; iii) [111] ordering (rocksalt $\mathrm{B}$ sublattice) parallel to the ferroelectric polarization, with R3m symmetry. Theoretical PZT isotropic, axial, and anisotropic chemical shifts are summarized in Table IV. [As mentioned, the axial and anisotropic components in Eq. (2) were calculated using the convention that the " $z$ " principal axis is that most nearly parallel to the $\mathrm{B}-\mathrm{O}-\mathrm{B}$ bond direction of the target $\mathrm{O}$ atom.] For comparison, results for $\mathrm{ST}, \mathrm{BT}, \mathrm{PT}$ and $\mathrm{PZ}$ are reproduced from Table III For each inequivalent target $\mathrm{O}$ atom in the above structural models, the corresponding 21 QM atom embedded cluster was used to calculate the chemical shielding tensor with both B3LYP and GGA 
TABLE IV: Calculated oxygen isotropic, axial and anisotropic components (ppm) of the chemical shift tensor for three PZT 50/50 structural models from Ref. 7. The notation B--O--B indicates O atoms with two equidistant nn B atoms, and B-O--B indicates an $\mathrm{O}$ atom with one short and one long nn B bond. For cluster-GGA results, numbers in parenthesis show the difference with GIPAW. For cluster-B3LYP results, numbers in square brackets show the difference with cluster-GGA values. For cases where $\delta_{\text {aniso }}=0$ by symmetry, this is indicated by a dash.

\begin{tabular}{|c|c|c|c|c|c|c|c|c|c|}
\hline & \multicolumn{3}{|c|}{$\delta_{\text {iso }}$} & \multicolumn{3}{|c|}{$\delta_{\mathrm{ax}}$} & \multicolumn{3}{|c|}{$\delta_{\text {aniso }}$} \\
\hline & \multicolumn{2}{|c|}{ Cluster } & \multirow{2}{*}{$\begin{array}{c}\text { GIPAW } \\
\text { GGA }\end{array}$} & \multicolumn{2}{|c|}{ Cluster } & \multirow{2}{*}{$\begin{array}{c}\text { GIPAW } \\
\text { GGA }\end{array}$} & \multicolumn{2}{|c|}{ Cluster } & \multirow{2}{*}{$\begin{array}{c}\text { GIPAW } \\
\text { GGA }\end{array}$} \\
\hline & B3LYP & GGA & & B3LYP & GGA & & B3LYP & GGA & \\
\hline ST (cubic) & $491[-3]$ & $494(-2)$ & 496 & $-144[-11]$ & $-133(12)$ & -145 & - & - & - \\
\hline BT (cubic) & $536[2]$ & $534(-41)$ & 575 & $-170[-16]$ & $-154(12)$ & -166 & - & - & - \\
\hline \multicolumn{10}{|l|}{$\mathrm{BT}(\mathrm{P} 4 \mathrm{~mm})$} \\
\hline Oax (Ti-O--Ti) & $591[20]$ & $571(-8)$ & 579 & $-207[-24]$ & $-183(3)$ & -186 & - & - & - \\
\hline Oeq (Ti--O--Ti) & $515[-1]$ & $516(-19)$ & 535 & $-155[-14]$ & $-140(7)$ & -147 & $-24[2]$ & $-26(15)$ & -41 \\
\hline \multicolumn{10}{|l|}{$\mathrm{PT}(\mathrm{P} 4 \mathrm{~mm})$} \\
\hline Oax (Ti-O--Ti) & $644[35]$ & $608(2)$ & 606 & $-257[-29]$ & $-228(8)$ & -236 & - & - & - \\
\hline Oeq (Ti-O--Ti) & $449[-8]$ & $457(10)$ & 447 & $-85[-10]$ & $-75(31)$ & -106 & $-22[7]$ & $-29(28)$ & -57 \\
\hline \multicolumn{10}{|l|}{ PZ(Pbam) } \\
\hline O1-4g (Z--O--Z) & $357[-10]$ & $367(12)$ & 355 & $-75[-5]$ & $-70(20)$ & -90 & $-33[1]$ & $-34(12)$ & -46 \\
\hline O1'-4g (Z--O--Z) & $346[-10]$ & $356(20)$ & 336 & $-65[-4]$ & $-61(21)$ & -81 & $-14[2]$ & $-16(11)$ & -27 \\
\hline $\mathrm{O} 2-8 \mathrm{i}(\mathrm{Z}-\mathrm{O}-\mathrm{-Z})$ & $355[-9]$ & $364(16)$ & 349 & $-81[-4]$ & $-77(14)$ & -91 & $-4[1]$ & $-5(2)$ & -7 \\
\hline O3-4f (Z--O--Z) & $324[-11]$ & $336(26)$ & 309 & $-47[-4]$ & $-43(9)$ & -52 & $-15[-1]$ & $-14(3)$ & -17 \\
\hline O4-4e (Z--O--Z) & $392[-6]$ & $399(-16)$ & 415 & $-137[-7]$ & $-130(36)$ & -166 & $-14[1]$ & $-15(11)$ & -26 \\
\hline \multicolumn{10}{|l|}{ PZT (P4mm) } \\
\hline O1(Zr--O--Zr) & $352[-9]$ & $361(33)$ & 328 & $-72[-6]$ & $-66(20)$ & -85 & $-16[-4]$ & $-12(-4)$ & -8 \\
\hline O2(Zr-O--Ti) & $398[7]$ & $391^{a}(-19)$ & 410 & $-126[-8]$ & $-118(17)$ & -135 & - & - & - \\
\hline O3(Ti--O--Ti) & $418[-18]$ & $436(9)$ & 427 & $-76[-11]$ & $-65(21)$ & -86 & $-59[4]$ & $-63(38)$ & -101 \\
\hline O4 (Ti-O--Zr) & $679[40]$ & $639(13)$ & 626 & $-266[-34]$ & $-232(14)$ & -246 & - & - & - \\
\hline \multicolumn{10}{|l|}{ PZT (P2mm) } \\
\hline O1(Zr--O--Zr) & $358[-9]$ & 367 (19) & 348 & $-80[-6]$ & $-74(22)$ & -96 & $-36[2]$ & $-38(20)$ & -59 \\
\hline $\mathrm{O} 2(\mathrm{Zr}-\mathrm{O}-\mathrm{-Zr})$ & $401[-4]$ & $405(-1)$ & 407 & $-124[-6]$ & $-118(18)$ & -137 & $-34[2]$ & $-36(9)$ & -44 \\
\hline O3(Ti--O--Ti) & $408[-11]$ & $419(24)$ & 395 & $-65[-8]$ & $-57(17)$ & -74 & $-6[5]$ & $-1(26)$ & -27 \\
\hline O4(Ti-O--Ti) & $668[39]$ & $629(12)$ & 617 & $-264[-32]$ & $-232(6)$ & -238 & $-49[3]$ & $-46(8)$ & -54 \\
\hline O5(Ti-O--Zr) & $400[-6]$ & $406(21)$ & 385 & $-81[-9]$ & $-72(20)$ & -92 & $-29[-3]$ & $-32(22)$ & -55 \\
\hline \multicolumn{10}{|l|}{ PZT (R3m) } \\
\hline O1(Zr-O--Ti) & $394[-7]$ & $401(0)$ & 401 & $-90[-6]$ & $-84(23)$ & -107 & $-3[1]$ & $-2(1)$ & -3 \\
\hline $\mathrm{O} 2(\mathrm{Ti}-\mathrm{O}-\mathrm{-Zr})$ & $466[-1]$ & $467(11)$ & 456 & $-127[-13]$ & $-114(21)$ & -135 & $-2[1]$ & $-1(-1)$ & -1 \\
\hline
\end{tabular}

${ }^{a}$ This cluster-GGA calculation used a smaller Pb cc-pVDZ basis, rather than cc-pVTZ, due to convergence difficulty with the larger basis.

exchange-correlation. Results from PBC-GIPAW with GGA exchange-correlation are also shown. Chemical shifts were determined using the corresponding values of $\sigma_{\text {ref }}^{\text {th. }}$ in Table III The GGA 21 QM atom embedded cluster and PBC-GIPAW RMS errors differ by $4 \mathrm{ppm}$. As seen in Tables III the $21 \mathrm{QM}$ atom embedded cluster chemical shifts are also in excellent agreement with those from the larger $65 \mathrm{QM}$ atom cluster.

PMN PBC-GIPAW calculations were based on a low symmetry $(3 \times 2 \times 2) 60$-atom perovskite supercell structure with relaxed internal coordinates, 37 with B-site cations arranged according to the random-site model 38 
X-ray patterns of well-annealed PMN samples indicate a homogeneous average structure, which exhibits rocksaltlike 1:1 B-site ordering, which is well described by the random-site model. Locally, the random-site model corresponds to B-site [111] planes, alternating between pure $\mathrm{Nb}$ and mixed $\mathrm{Nb} / \mathrm{Mg}$ layers. Thus there are twice as many $\mathrm{Nb}-\mathrm{O}-\mathrm{Mg}$ as $\mathrm{Nb}-\mathrm{O}-\mathrm{Nb} \mathrm{O}$-sites in the 60 -atom supercell, since the $\mathrm{Mg}$ atoms all reside in the mixed [111] planes. Similarly, there are no Mg-O-Mg O-sites. Unlike earlier models, the random-site model satisfies charge neutrality locally. The previously accepted space-charge model was based on the apparent inability to fully anneal samples,$\underline{38}$ The 60 -atom perovskite supercell structure,,$\underline{37}$ used in the present calculations, is consistent with the random-site model. Grinberg et al $\stackrel{37}{=}$ found good agreement between this relaxed 60-atom supercell structure and pair distribution functions (PDFs) obtained by neutron scattering experiments $\underline{\underline{39}}$ This indicates that this structural model reasonably represents the local structure in PMN. Calculations for PMN were done only with PBC-GIPAW, and the results are shown in Table $\mathrm{V}$.

\section{DISCUSSION}

As previously noted in Ref. 8, there is a large anisotropy between the $\sigma_{x, y}$ and $\sigma_{z}$ principal values in Table [I] The $\sigma_{x, y}$ principal values are large and negative (deshielded), while $\sigma_{z}$ values tend to be considerably smaller and positive (shielded). (As mentioned, the " $z$ " principal axis is identified as that most closely aligned with the $\mathrm{B}-\mathrm{O}-\mathrm{B}$ bond direction of the target $\mathrm{O}$ atom.) The present calculations show that this anisotropy is also found in PZT and PMN and is reflected in the large $\delta_{a x}$ values (Eq. 2) in Tables IV and V

As shown in Ref. 8 for prototypical perovskites, the large $\delta_{a x}$ values are due to paramagnetic contributions to $\sigma_{x, y}$ from virtual transitions between $\mathrm{O}(2 \mathrm{p})$ and unoccupied $\mathrm{B}(n \mathrm{~d})$ states. The p-d hybridization contributes predominantly to $\sigma_{x, y}$, due to $\mathrm{O}$ atoms having only two nearest neighbors in perovskites, with linearly arranged B-O-B structural units. As the B-O-B bond distances vary, large variations in the chemical shielding can occur, resulting in a strong dependence on $r_{\mathrm{s}}$. We find a similar dependence for the PZT and PMN alloy systems.

\section{A. PZT}

Figure 1 plots PZT isotropic and axial chemical shifts as a function of $r_{\mathrm{s}}$, the shortest B-O bond length of the targeted $\mathrm{O}$ atom. The B3LYP $21 \mathrm{QM}$ atom cluster results from Table IV are plotted. For comparison, results for ST, BT, PT, and PZ and from experiment are also shown $\stackrel{8}{*}$ The dashed straight lines are the linear fits to the calculated results for these prototypical perovskites, taken from Ref. 8. A plot of PBC-GIPAW results from Table IV (not shown) is very similar, which is consistent with the generally good agreement of the chemical shift results in the Table between the two calculational approaches. A nearly linear dependence on $r_{\mathrm{s}}$ (with slope $\sim 850 \mathrm{ppm} / \AA$ ) is seen in both $\delta_{\text {iso }}$ and $\delta_{\mathrm{ax}}$, across all the systems studied. The axial shift is plotted as $2 \delta_{\text {ax }}=\delta_{z}-\delta_{\text {iso }}$ (Eq. (2) to emphasize that the linear dependence is largely due to $\delta_{x, y}$, while $\delta_{z}$ has a much weaker dependence on $r_{\mathrm{s}}$, as previously noted for

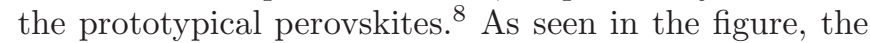
calculated PZT 50/50 results follow the same trends as in Ref. 8 .

$\mathrm{Pb}\left(\mathrm{Zr}_{1-x} \mathrm{Ti}_{x}\right) \mathrm{O}_{3} \quad \mathrm{NMR}{ }^{17} \mathrm{O}$ magic angle spinning (MAS) central peak spectra were presented by Baldwin et $a l^{\underline{4}}$ for a range of concentrations $x$. Tabulated chemical shifts were given only for the endpoint PT and PZ compounds (reproduced here in Table III), whose spectra consist of well-defined narrow peaks. MAS removes broadening due to chemical shift anisotropy in powder samples, but only partly averages secondorder quadrupolar broadening. The narrow peaks in the endpoint PT and PZ compounds indicate that electric field gradients (EFGs) at the ${ }^{17} \mathrm{O}$ nuclei are small $\underline{4}$ This is consistent with first-principles calculations of $\mathrm{O}$ EFGs $\stackrel{7.36}{=}$ Indeed, the ${ }^{17} \mathrm{O}$ peak positions are within a few ppm of the experimental isotropic chemical shifts. $\underline{4}$ There are two inequivalent $\mathrm{O}$ atoms in $\mathrm{PT}$, two "equatorial" $\mathrm{O}_{\mathrm{eq}}$ (which has two equidistant $\mathrm{nn}$ Ti atom) and one "axial" $\mathrm{O}_{\mathrm{ax}}$ atom (which has one short and one long Ti-O bond). A narrow peak at $443 \mathrm{ppm}$ has twice the (integrated) relative intensity of the peak at $647 \mathrm{ppm}$, and these were assigned to the $\mathrm{O}_{\text {eq }}$ and $\mathrm{O}_{\mathrm{ax}}$ atoms, respectively. These are in good agreement with the calculated results in Table III. The B3LYP calculation accurately reproduces this splitting, while GGA underestimates it by $\simeq 45 \mathrm{ppm}$. In PZ, there are five inequivalent $\mathrm{O}$ sites, which corresponds to five MAS peaks, centered near $350 \mathrm{ppm}$, within $\pm \sim 40 \mathrm{ppm}$. Measured and calculated values in Table III are in good agreement.

At intermediate Ti and $\mathrm{Zr}$ concentrations, the spectra in Fig. 3 of Ref. 4 show that only a few of the narrow endpoint peaks persist. As $\mathrm{Zr}$ is added to $\mathrm{PT}$, the narrow $647 \mathrm{ppm}$ PT peak decreases quickly in intensity. It can no longer be observed in the $x=0.55$ sample. A broad feature, between about 350 and $450 \mathrm{ppm}$ is fully developed near $x \simeq 0.50$, with narrower embedded features at $\simeq 370$ and $430 \mathrm{ppm}$. This broad feature distribution of inequivalent $\mathrm{O}$-sites in the disordered PZT solid solution samples. The $430 \mathrm{ppm}$ feature, which is close to the PT $\mathrm{O}_{\text {eq }} 447 \mathrm{ppm}$ peak, is observed to persist down to $25 \% \mathrm{Ti}$ concentration. Baldwin et al 年 assign the $430 \mathrm{ppm}$ feature to a site similar to that of the $\mathrm{PT} \mathrm{O}_{\text {eq }}$ atom, i.e. a locally $\mathrm{Ti}-\mathrm{O}_{\mathrm{eq}}-\mathrm{Ti}$ (undimerized) chain-like configuration. They further suggest that this peak could indicate $\mathrm{Ti}$ clustering on a spatial scale of at least two unit cells in PZT.

Our results suggest an alternative explanation for the persistence of the observed $430 \mathrm{ppm}$ feature. Our calcu- 


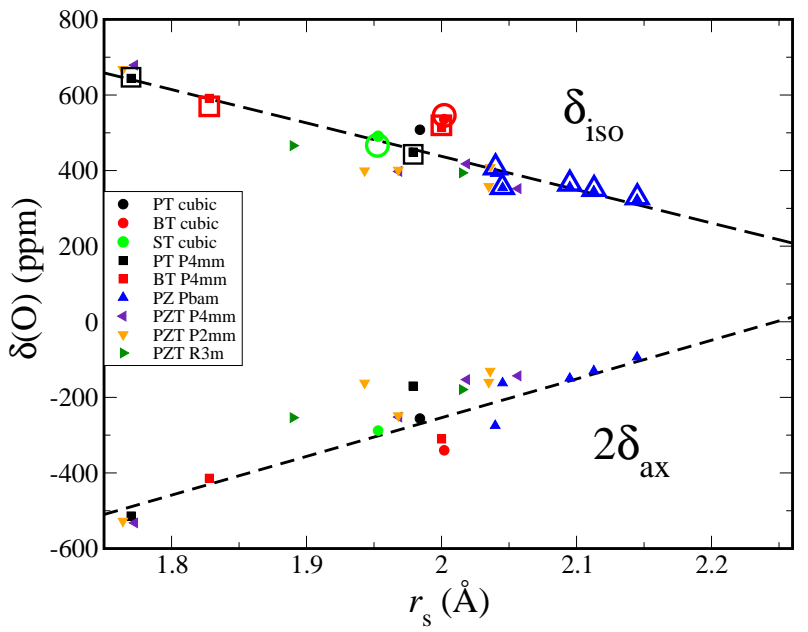

FIG. 1: (Color online) Calculated PZT oxygen isotropic $\delta_{\text {iso }}$ and axial $2 \delta_{\text {ax }}$ chemical shifts (B3LYP 21 QM atom cluster values from Tables (IV), plotted as a function of $r_{\mathrm{s}}$, the shortest $\mathrm{B}-\mathrm{O}$ bond length of the targeted $\mathrm{O}$ atom. For comparison, calculated results for ST, BT, PT, and PZ and from experiment (hollow symbols) are also shown $\stackrel{8}{-}$ The dashed straight lines are linear fits to calculated values for ST, BT, PT, and PZ from Ref. 8.

lations show similar chemical shifts for a range of B-O-B' environments, with $r_{\mathrm{s}}$ ranging between $\sim 2.0-2.1 \AA$ in all the PZT 50/50 structural models, as seen in TableIV and Fig. 1 Thus Ti clustering need not be invoked to explain the persistence of the $430 \mathrm{ppm}$ feature in the measured spectra.

The apparent disappearance, at intermediate concentrations, of the $647 \mathrm{ppm}$ peak does, however, indicate a reduced occurrence of a PT-like $\mathrm{O}_{\mathrm{ax}}$ site with a short $1.77 \AA$ Ti-O bond. We find such a site only in the relaxed P4mm and P2mm PZT 50/50 simulations. The P4mm and $\mathrm{P} 2 \mathrm{~mm}$ models have [100] type B-site ordering. The absence of the $647 \mathrm{ppm}$ peak in the measurements indicates that local occurrences of [100] type B-site ordering are rare. Instead, the lack of the $647 \mathrm{ppm}$ peak in our R3m PZT 50/50 simulations suggests that local rocksaltlike B-site ordering is more prevalent in disordered PZT. This conclusion is also supported by the R3m structural model having the lowest total energy of all the structural models by $\sim 23 \mathrm{mRy} \underline{\underline{7}}$ Moreover, the calculated $\mathrm{Ti}$ EFG's for 50/50 R3m were significantly smaller and in better agreement with measured values, than the other B-site orderings $\stackrel{\underline{7}}{\underline{7}}$

Baldwin et al $\underline{\underline{4}}$ also remark a narrow $287 \mathrm{ppm}$ peak that appears in their $x=0.48$ sample. This peak is broader in $x=0.55$ and $x=0.25$ samples and is absent in PZ. These authors note that $x=0.48$ is the composition corresponding to the morphotropic phase boundary (MPB) and conjecture that the $287 \mathrm{ppm}$ peak evidences a new oxygen environment in a distinct crystalline monoclinic phase, which has been suggested to bridge the MPB $\stackrel{\underline{4}}{\underline{4}}$ They suggest that the new environment at

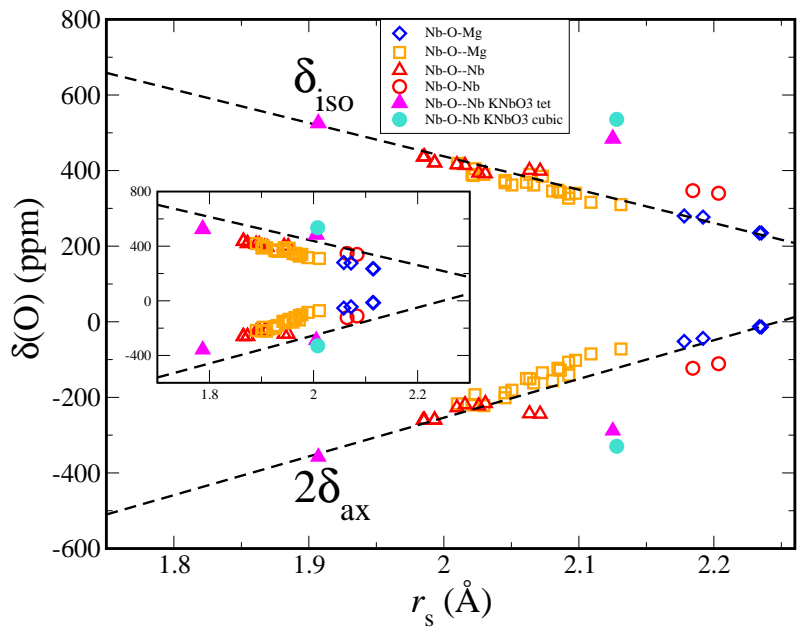

FIG. 2: (Color online) GIPAW calculated PMN oxygen isotropic $\delta_{\text {iso }}$ and axial $2 \delta_{\text {ax }}$ chemical shifts, plotted as a function of $r_{\mathrm{s}}$. The PMN chemical shifts are from Table $\mathrm{V}$ and the symbols indicate the B-O- $\mathrm{B}^{\prime}$ configuration, using the convention in that table. For comparison, results for cubic and tetragonal $\mathrm{KNbO}_{3}$ are also shown. The dashed lines are the same as that in Fig. 1. As plotted, the $r_{\mathrm{s}}$ values have been increased by $0.12 \AA$ (see text). The unshifted values are shown in the inset.

$x=0.48$ is a $\mathrm{Ti}-\mathrm{O}-\mathrm{Zr}$ site, which becomes ordered in the crystallographic sense as the long-range order of the monoclinic phase is established. As indicated by Fig. 1. isotropic chemicals shifts near $287 \mathrm{ppm}$ are associated with large $r_{\mathrm{s}} \simeq 2.15 \AA$. As most $\mathrm{Zr}-\mathrm{O}$ bond lengths are larger than those of $\mathrm{Ti}-\mathrm{O}$ in the $\mathrm{PZ}$ and PZT 50/50 structural models, the $287 \mathrm{ppm}$ feature could also be attributed to $\mathrm{Zr}-\mathrm{O}-\mathrm{Zr}$ sites with bond lengths distributed near $\simeq 2.15 \AA$.

\section{B. PMN}

PMN, by contrast with PZT, is a heterovalent 2:1 mixture of the $\mathrm{Nb}^{5+}$ transition metal cation and the $\mathrm{Mg}^{2+}$ alkaline earth cation. Figure 2 plots $\mathrm{Pb}\left(\mathrm{Mg}_{1 / 3} \mathrm{Nb}_{2 / 3}\right) \mathrm{O}_{3}$ (PMN) isotropic and axial chemical shifts (GIPAW results from Table $\mathrm{V}$ as a function of $r_{\mathrm{s}}$, the shortest B-O bond length of the targeted $\mathrm{O}$ atom. As plotted, the PMN $r_{\mathrm{s}}$ values, have been increased by $0.12 \AA$ for both $\delta_{\text {iso }}$ and $\delta_{\mathrm{ax}}$, as further discussed below. The unshifted values are plotted in the inset. The dashed lines are the same as that in Fig. 1. With the $0.12 \AA$ rigid shift for all O-sites, the PMN chemical shifts are seen to follow the same linear trend as in the homovalent B-site systems in Fig. 1. For Nb-O-Mg coordinated O-atoms, $r_{\mathrm{s}}$ is taken as the $\mathrm{Nb}-\mathrm{O}$ bond length, since the hybridization mechanism does not apply to $\mathrm{Mg}$, which has no low-lying unoccupied d-states. (As mentioned, the linear dependence is due to paramagnetic contributions to $\delta_{\mathrm{xy}}$ from virtual transitions between $\mathrm{O}(2 \mathrm{p})$ and unoccupied $\mathrm{B}(n \mathrm{~d})$ 
states.) We are not aware of published $\mathrm{NMR}^{17} \mathrm{O}$ spectra for PMN.

Although the linear dependence of $\delta_{\text {iso }}$ and $\delta_{\text {ax }}$ in Fig.2 have the same slope as the homovalent B-site systems, a $0.12 \AA r_{\mathrm{s}}$ offset is needed for the PMN values to fall on the same line. As mentioned, the linear dependence reflects variations in the magnitude of the paramagnetic $\mathrm{O}(2 \mathrm{p})$ $\mathrm{Nb}(4 \mathrm{~d})$ hybridization contributions to the $\delta_{x, y}$ principal values. This indicates that the effective $r_{\mathrm{s}}$ is controlled by the spatial extent of the paramagnetic screening currents. The $\mathrm{Nb}^{5+}$ cation could be expected to modify the spatial extent of these currents, compared to the $\mathrm{B}^{4+}$ cations in the homovalent systems, due to the larger electrostatic attraction of the $\mathrm{Nb}^{5+}$ cation. The $0.12 \AA$ offset in PMN renormalizes, in effect, the strength of the $\mathrm{O}(2 \mathrm{p})-\mathrm{Nb}(4 \mathrm{~d})$ hybridization. This observation would appear to indicate that for these systems the $r_{\mathrm{s}}$-dependence of $\delta_{x, y}$ is given by $\delta_{x, y}=m\left(r_{\mathrm{s}}-r_{0}\right)$, where the slope $m$ is nearly the same for all transition-metal coordinated $\mathrm{O}$ atoms in perovskites, while the intercept $r_{0}$ depends on other factors, such as the ionic charge of the nearest neighbor cation. To further examine this, we performed GIPAW calculations for cubic and tetragonal $\mathrm{KNbO}_{3} \stackrel{40}{\underline{40}}$ which are also plotted in Fig. 2. While the tetragonal axial $\mathrm{O}$ values (short $\mathrm{Nb}-\mathrm{O} r_{\mathrm{s}}$ ) are consistent with the linear trend, the $\mathrm{KNbO}_{3}$ tetragonal equatorial and cubic $\mathrm{O}$ results (two equidistant $\mathrm{Nb-O}$ bonds) show significant deviations. [Similar deviations occur in Fig. 1 for the tetragonal equatorial and cubic O BT results. We note that both $\mathrm{K}$ and $\mathrm{Ba}$ have larger crystalline ionic Shannon $\stackrel{41}{\underline{1}}$ radii, 1.78 and $1.75 \AA$, respectively, than $\mathrm{Sr}$ or $\mathrm{Pb}, 1.58$ and $1.63 \AA$, respectively.] Smaller deviations are also seen in the two largest $r_{\mathrm{s}}$ values for the PMN oxygen atoms with two nearly equidistant $\mathrm{Nb}$ atoms, but for somewhat larger values of $r_{\mathrm{s}}$ than in $\mathrm{KNbO}_{3}$. In the case of heterovalent B-site perovskites, the covalency of A-site atoms could also be important, as indicated by the smaller deviations, for long $r_{\mathrm{s}}$, in PMN compared to $\mathrm{KNbO}_{3}$. These observations warrant further investigation to clarify these issues.

$\mathrm{PMN}$ is an end-point of the solid-solution series (x) $\mathrm{PbTiO}_{3}-(1-x) \mathrm{Pb}\left(\mathrm{Mg}_{1 / 3} \mathrm{Nb}_{2 / 3}\right) \mathrm{O}_{3} \quad$ (PMN-PT). In the 60-atom PMN structural model, there are no Nb$\mathrm{O}$ bonds as small as the short Ti-O $\mathrm{O}_{\mathrm{ax}}$ bond $\simeq 1.7 \AA$ in $\mathrm{PT}$, which is associated with its high degree of tetragonality $(c / a=1.065)$. This short bond corresponds to the large $\delta_{\text {iso }} \simeq 640 \mathrm{ppm}$, which is also seen in the $\mathrm{P} 4 \mathrm{~mm}$ and P2mm PZT structural models, both of which also show a high degree of tetragonality. The tetragonality of PMN-PT decreases as the Ti concentration is reduced from PT-rich compositions, and the average symmetry switches from tetragonal to rhombohedral at the mor- photropic phase boundary (MPB) $x \simeq 0.35$. The largest piezoelectric response is typically achieved at concentrations near the MPB $\stackrel{42}{=}$ Polarization rotation has been proposed as the origin of the large piezoelectric response at the MPB, via intermediate monoclinic phases $\underline{43-45}$ where the tetragonality increases as the polarization rotates from [111] to [100] (pseudocubic) directions, with applied electric field along a pseudocubic axis. Increased tetragonality, compared to $\mathrm{PT}$, has been seen in some other perovskite based solid solutions, such as some Bi based materials $\underline{46,47}$ The present calculations indicate that ${ }^{17} \mathrm{O}$ NMR chemical shift measurements could be a useful probe in this regard, as increased tetragonality is accompanied by shortened transition-metal/oxygen bonds.

\section{SUMMARY}

First-principles oxygen NMR chemical shift tensors were calculated for PZT and PMN, which are representative, respectively, of homovalent and heterovalent perovskite-structure B-site alloys. Quantum chemistry methods for embedded clusters and the GIPAW method for periodic boundary conditions were used. Results from both methods are in good agreement for PZT and prototypical perovskites. PMN results were obtained using only GIPAW. Both isotropic and axial chemical shift components were found to vary approximately linearly as a function of the nearest-distance transitionmetal/oxygen bond length, $r_{\mathrm{s}}$. Using these results, we argue against Ti clustering in PZT, as conjectured from recent ${ }^{17} \mathrm{O}$ NMR measurements. Our findings indicate that ${ }^{17} \mathrm{O}$ NMR measurements, coupled with first-principles calculations, can be an important probe of local structure in complex perovskite solid solutions.

\section{ACKNOWLEDGMENTS}

This research was supported by Office of Naval Research grants N00014-08-1-1235 and N00014-09-1-0300. DLP acknowledges partial support from a Virginia Space Grant Consortium Graduate Research Fellowship. Support for computations was provided in part by National Science Foundation TeraGrid resources at the National Center for Supercomputing Applications (NCSA) under grant number TG-DMR100024. Additional computational resources were provided by the Center for Piezoelectric by Design. We acknowledge useful discussions with Gina Hoatson and Robert L. Vold.
1 M. Dawber, K. Rabe, and J. Scott Rev. Mod. Phys., vol. 77, no. 4, p. 1083, 2005.
2 J. Scott Science, vol. 315, p. 954, 2007.

3 D. H. Zhou, G. L. Hoatson, R. L. Vold, and F. Fayon Phys. 
Rev. B, vol. 69, p. 134104, 2004.

4 A. Baldwin, P. A. Thomas, and R. Dupree J. Phys.: Condens. Matter, vol. 17, p. 7159, 2005.

${ }^{5}$ M. Vijayakumar, G. L. Hoatson, and R. L. Vold Phys. Rev. $B$, vol. 75, p. 104104, 2007.

${ }^{6}$ R. Blinc, V. V. Laguta, B. Zalar, M. Itoh, and H. Krakauer J. Phys.: Condens. Matter, vol. 20, p. 085204, 2008.

7 D. Mao, E. J. Walter, H. Krakauer, and Z. Wu Phys. Rev. B, vol. 76, p. 014105, 2007.

8 D. L. Pechkis, E. J. Walter, and H. Krakauer J. Chem. Phys., vol. 131, no. 18, p. 184511, 2009.

9 M. J. Frisch, G. W. Trucks, H. B. Schlegel, and et al., "Gaussian 98, Revision A.11.4." Gaussian, Inc., Pittsburgh PA, 2002.

${ }^{10}$ M. J. Frisch, G. W. Trucks, H. B. Schlegel, and et al., "Gaussian 03, Revision C.02." Gaussian, Inc., Wallingford, CT, 2004.

11 M. J. Frisch, G. W. Trucks, H. B. Schlegel, and et al., "Gaussian 09, Revision A.02,." Gaussian, Wallingford CT, 2009.

12 T. Helgaker, M. Jaszunski, and K. Ruud Chem. Rev., vol. 99, no. 1, p. 293, 1999.

13 M. Kaupp, M. Bühl, and V. Malkin, Calculation of NMR and EPR Parameters: Theory and Applications. WileyVCH, Weinnheim, 2004.

14 J. Vaara Phys. Chem. Chem.l Phys., vol. 9, p. 5399, 2007.

${ }^{15}$ K. L. Schuchardt, B. T. Didier, T. Elsethagen, L. Sun, V. Gurumoorthi, J. Chase, J. Li, and T. L. Windus, "Basis set exchange: A community database for computational sciences," J. Chem. Inf. Model., vol. 47, no. 3, pp. 10451052, 2007.

16 C. G. V. de Walle and P. E. Blöchl Phys. Rev. B, vol. 47, p. $4244,1993$.

17 L. Truflandier, M. Paris, and F. Boucher Phys. Rev. B, vol. 76, p. 035102, 2007.

18 D. S. Middlemiss, F. Blanc, C. J. Pickard, and C. P. Grey J. Magn. Reson., vol. 204, p. 1, 2010.

19 R. F. Schneider J. Chem. Phys., vol. 48, p. 4905, 1968.

${ }^{20}$ F. Anet and D. J. O'Leary Concepts in Magn. Reson, vol. 3, p. 193, 1991.

21 J. F. Baugher, P. C. Taylor, T. Oja, and P. J. Bray J. Chem. Phys., vol. 50, p. 4914, 1969.

22 T. Charpentier, S. Ispas, M. Profeta, F. Mauri, and C. J. Pickard J. Phys. Chem. B, vol. 108, p. 4147, 2004.

${ }^{23}$ M. K. Klintenberg, S. E. Derenzo, and M. J. Weber Comput. Phys. Commun., vol. 131, p. 120, 2000.

24 N. W. Winter, R. M. Pitzer, and D. K. Temple J. Chem. Phys., vol. 86, p. 3549, 1987.

${ }^{25}$ T. A. Keith and R. F. W. Bader Chem. Phys. Lett., vol. 210 , p. 223, 1993.

26 J. R. Cheeseman, G. W. Trucks, T. A. Keith, and M. J. Frisch J. Chem. Phys., vol. 104, p. 5497, 1996.
27 A. D. Becke J.Chem. Phys, vol. 98, p. 5648, 1993.

${ }^{28}$ K. Burke, J. P. Perdew, and Y. Wang, Electronic Density Functional Theory: Recent Progress and New Directions. Plenum, 1998.

29 J. P. Perdew, K. Burke, , and M. Ernzerhof Phys. Rev. Lett., vol. 77, p. 3865, 1996.

30 P. Giannozzi, S. Baroni, N. Bonini, M. Calandra, R. Car, C. Cavazzoni, D. Ceresoli, G. L. Chiarotti, M. Cococcioni, I. Dabo, A. Dal Corso, S. de Gironcoli, S. Fabris, G. Fratesi, R. Gebauer, U. Gerstmann, C. Gougoussis, A. Kokalj, M. Lazzeri, L. Martin-Samos, N. Marzari, F. Mauri, R. Mazzarello, S. Paolini, A. Pasquarello, L. Paulatto, C. Sbraccia, S. Scandolo, G. Sclauzero, A. P. Seitsonen, A. Smogunov, P. Umari, and R. M. Wentzcovitch J. Phys.: Condens. Matter, vol. 21, p. 395502, 2009.

31 H. J. Monkhorst and J. D. Pack Phys. Rev. B, vol. 13, p. $5188,1976$.

32 N. Troullier and J. L. Martins Phys. Rev. B, vol. 43, p. 1993, 1991.

33 D. R. Hamann Phys. Rev. B, vol. 40, p. 2980, 1989.

${ }^{34}$ R. E. Wasylishen and D. Bryce J. Chem. Phys., vol. 117, p. 10061, 2002.

35 S. C. Abrahams and J. L. Bernstein J. Chem. Phys., vol. 55, p. 3206, 1971.

36 M. D. Johannes and D. J. Singh Phys. Rev. B, vol. 71, p. 212101, 2005.

37 I. Grinberg and A. M. Rappe Phys. Rev. B, vol. 70, p. 220101, 2004.

38 P. K. Davies and M. A. Akbas J. Phys. Chem. Solids, vol. 61 , p. 159, 2000.

39 T. Egami, W. Dmowski, M. Akbas, and P. K. Davies, "Local structure and polarization in $\mathrm{Pb}$ containing ferroelectric oxides," vol. 436, p. 1, AIP, 1998.

40 C.-Z. Wang, R. Yu, and H. Krakauer Phys. Rev. B, vol. 54, p. 11161, 1996.

41 R. D. Shannon, "Revised effective ionic radii and systematic studies of interatomic distances in halides and chalcogenides," Acta Crystallographica Section A, vol. 32, pp. 751-767, Sep 1976.

42 S.-E. Park and T. R. Shrout J. Appl. Phys., vol. 82, p. 1804, 1997.

43 B. Noheda and D. E. Cox Phase Transitions, vol. 79, p. 5, 2006. (See references within).

${ }^{44}$ H. Fu and R. Cohen Nature, vol. 403, p. 281, 2000.

45 Z. Wu and H. Krakauer Phys. Rev. B, vol. 68, p. 014112, Jul 2003.

46 M. R. Suchomel and P. K. Davies Appl. Phys. Lett., vol. 86, p. 262905, 2005.

47 D. M. Stein, M. R. Suchomel, and P. K. Davies Appl. Phys. Lett., vol. 89, p. 132907, 2006. 
TABLE V: GIPAW calculated oxygen isotropic, axial and anisotropic components (ppm) of the chemical shift tensor for PMN. A 60-atom supercell with relaxed internal coordinates was used ${ }^{37}$ The B-O-- $\mathrm{B}^{\prime}$ bond notation is the same as in Table IV bond lengths are categorized as equidistant if they differ by less than $0.05 \AA$.

\begin{tabular}{|c|c|c|c|c|c|}
\hline & $\begin{array}{c}\mathrm{Nb}-\mathrm{O} \\
\AA\end{array}$ & $\begin{array}{c}\mathrm{B}^{\prime}-\mathrm{O} \\
\AA \\
\AA\end{array}$ & $\delta_{\text {iso }}$ & $\delta_{\mathrm{ax}}$ & $\delta_{\text {aniso }}$ \\
\hline \multicolumn{6}{|l|}{$\mathrm{Nb}-\mathrm{O}-\mathrm{Mg}$} \\
\hline & 2.11 & 2.09 & 235 & -6 & -5 \\
\hline & 2.11 & 2.09 & 235 & -7 & -2 \\
\hline & 2.06 & 2.08 & 280 & -26 & -47 \\
\hline & 2.07 & 2.09 & 277 & -22 & -43 \\
\hline \multicolumn{6}{|l|}{$\mathrm{Nb}-\mathrm{O}-\mathrm{-Mg}$} \\
\hline & 1.89 & 2.06 & 419 & -109 & -27 \\
\hline & 1.90 & 2.07 & 405 & -97 & -11 \\
\hline & 1.90 & 2.09 & 390 & -110 & -25 \\
\hline & 1.90 & 2.11 & 387 & -110 & -31 \\
\hline & 1.91 & 2.11 & 390 & -111 & -83 \\
\hline & 1.93 & 2.08 & 362 & -91 & -7 \\
\hline & 1.93 & 2.09 & 368 & -94 & -10 \\
\hline & 1.93 & 2.10 & 374 & -100 & -83 \\
\hline & 1.94 & 2.13 & 369 & -75 & -36 \\
\hline & 1.94 & 2.18 & 390 & -75 & -17 \\
\hline & 1.95 & 2.11 & 362 & -81 & -11 \\
\hline & 1.95 & 2.18 & 385 & -67 & -18 \\
\hline & 1.96 & 2.05 & 346 & -78 & -29 \\
\hline & 1.96 & 2.12 & 348 & -61 & -44 \\
\hline & 1.97 & 2.05 & 338 & -71 & -36 \\
\hline & 1.97 & 2.10 & 343 & -64 & -11 \\
\hline & 1.97 & 2.13 & 327 & -53 & -13 \\
\hline & 1.98 & 2.06 & 340 & -51 & -47 \\
\hline & 1.99 & 2.13 & 316 & -43 & -7 \\
\hline & 2.01 & 2.06 & 310 & -36 & -39 \\
\hline \multicolumn{6}{|l|}{$\mathrm{Nb}-\mathrm{O}--\mathrm{Nb}$} \\
\hline & 1.86 & 2.23 & 436 & -130 & -21 \\
\hline & 1.87 & 2.14 & 421 & -130 & -3 \\
\hline & 1.87 & 2.14 & 421 & -129 & -8 \\
\hline & 1.87 & 2.23 & 437 & -130 & -18 \\
\hline & 1.89 & 2.20 & 416 & -114 & -27 \\
\hline & 1.90 & 2.20 & 414 & -110 & -24 \\
\hline & 1.91 & 2.11 & 394 & -111 & -23 \\
\hline & 1.91 & 2.11 & 392 & -108 & -18 \\
\hline & 1.94 & 2.03 & 401 & -122 & -8 \\
\hline & 1.95 & 2.01 & 399 & -122 & -11 \\
\hline \multicolumn{6}{|l|}{$\mathrm{Nb}-\mathrm{O}-\mathrm{Nb}$} \\
\hline & 2.06 & 2.09 & 347 & -62 & -9 \\
\hline & 2.08 & 2.08 & 340 & -56 & -11 \\
\hline
\end{tabular}

